\title{
Insect Management for Legumes (Beans, Peas) ${ }^{1}$
}

\section{S. E. Webb²}

Many different insects attack leguminous vegetables. Aphids damage terminals, whiteflies feed on sap and transmit bean golden mosaic virus, and caterpillars like bean leafroller and beetles feed on leaves. Flower thrips feed in blossoms and stink bugs, corn earworm, and leaffooted bugs damage seeds and pods.

Snap beans are becoming an important crop for Florida. Southern peas (a bean) are common in north Florida. Many other types of beans are grown on a small scale. Increasingly, newer pesticides are being registered for the entire crop group, including legumes such as pigeon pea, yardlong bean, swordbean, and crowder pea. Labels for individual insecticides should be consulted to see if they are labeled for all types of beans.

According to already published guidelines for snap beans (Pernezney et al., 2003) management practices should include scouting twice a week for insect pests in at least one location for every 2.5 acres. More sites should be chosen in small fields (less than 20 acres). A map of the field should be drawn so that pest counts can be connected to a particular section of the field for future reference.

A sample is a 3 section of row. Whiteflies can be estimated by turning over several leaves in the section and counting the number of adults. Terminals should be examined for the presence of aphids. For other pests, a three by threecloth is placed on the ground and the bean plants shaken over it. Insects that fall on the cloth can be identified and counted. The growth stage of the plant and an estimate of defoliation should be recorded. Snap beans can tolerate up to $20 \%$ defoliation before pod set and $10 \%$ after pod set.

A systemic insecticide (a neonicotinoid) should be applied at planting to control aphids and whiteflies. Later in the season, when the effects of the systemic wear off, an insect growth regulator for whiteflies may be applied. Because it is the pod that is sold, damage to this part of the plant is the most serious. An insecticide appropriate for the pests present should be applied at pinpod. At least one more application may be needed before harvest.

1. This document is ENY-465 (IG151), one of a series of the Entomology \& Nematology Department, Florida Cooperative Extension Service, Institute of Food and Agricultural Sciences, University of Florida. Published: July 2002. Revised: August 2005. For more publications related to horticulture/agriculture, please visit the EDIS Website at http://edis.ifas.ufl.edu/. For more information about nematodes, arthropods, and other invertebrates, please visit the Entomology \& Nematology Department website at http://entnemdept.ifas.ufl.edu/.

2. S. E. Webb, associate professor, Entomology and Nematology Department, Cooperative Extension Service, Institute of Food and Agricultural Sciences, University of Florida, Gainesville, 32611-0640.

The use of trade names in this publication is solely for the purpose of providing specific information. UF/IFAS does not guarantee or warranty the products named, and references to them in this publication does not signify our approval to the exclusion of other products of suitable composition. All chemicals should be used in accordance with directions on the manufacturer's label. Use pesticides safely. Read and follow directions on the manufacturer's label. 
For the organic grower, a number of

OMRI-listed insecticides have been listed in the table (see the Notes column).

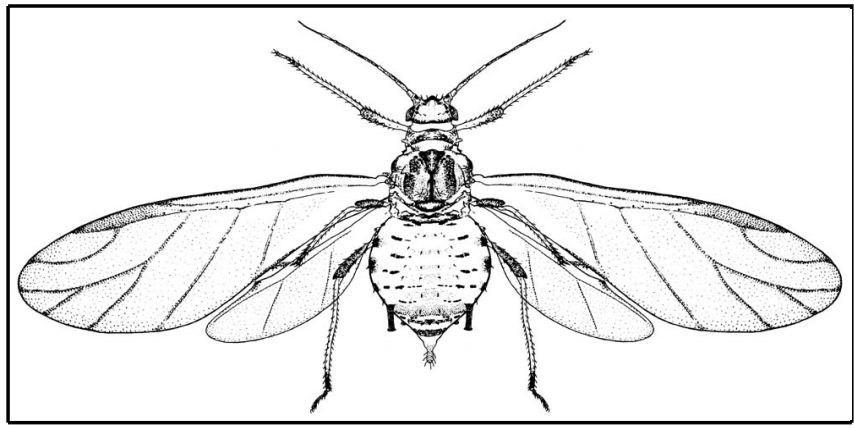

Figure 1. Cowpea aphid, Aphis craccivora Koch. Credits: John L. Capinera, University of Florida

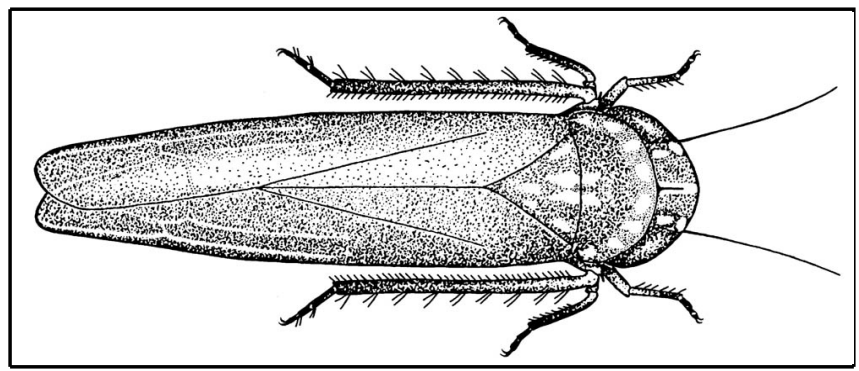

Figure 2. Potato leafhopper, Empoasca fabae. Credits: John L. Capinera, University of Florida

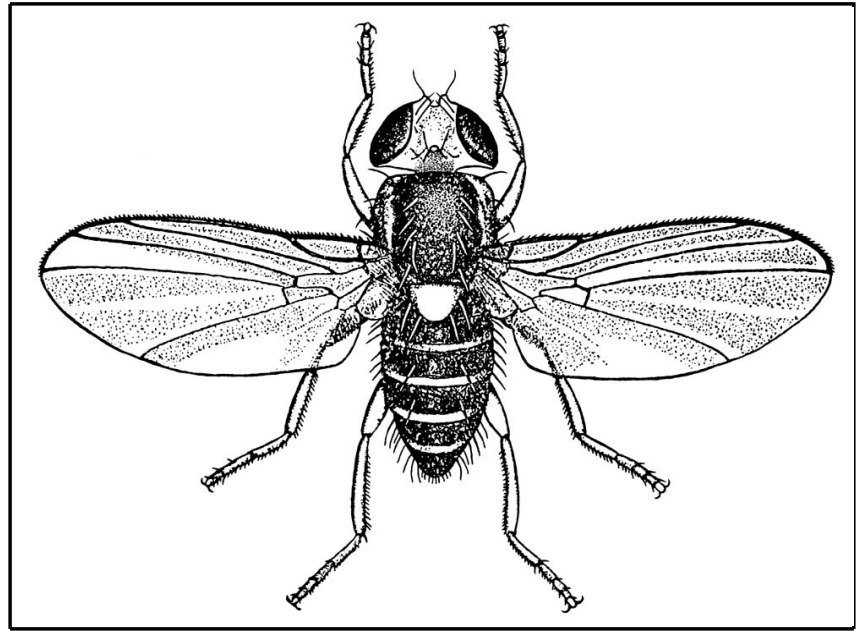

Figure 3. American serpentine leafminer, Liriomyza trifolii (Burgess) Credits: John L. Capinera, University of Florida

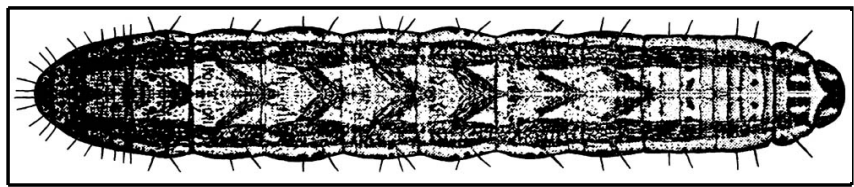

Figure 4. Granulate cutworm larva, Feltia subterranea (F.). Credits: USDA

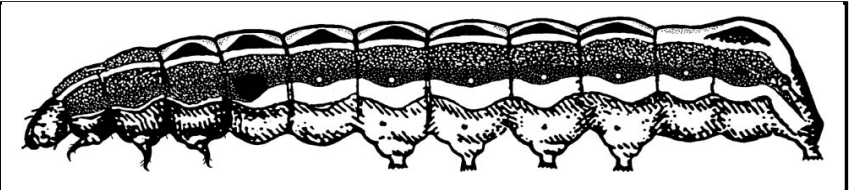

Figure 5. Southern armyworm larva, Spodoptera eridania (Cramer) Credits: John L. Capinera, University of Florida

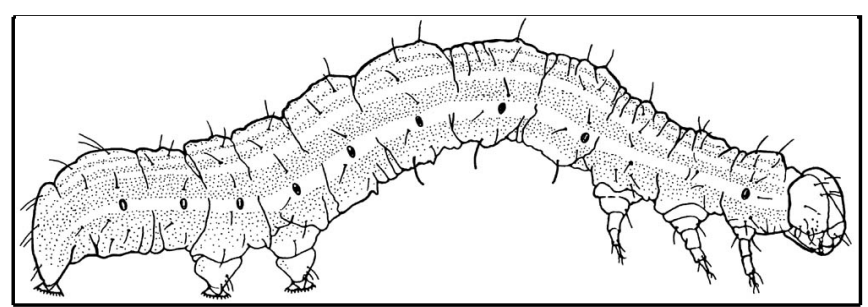

Figure 6. Cabbage looper larva.

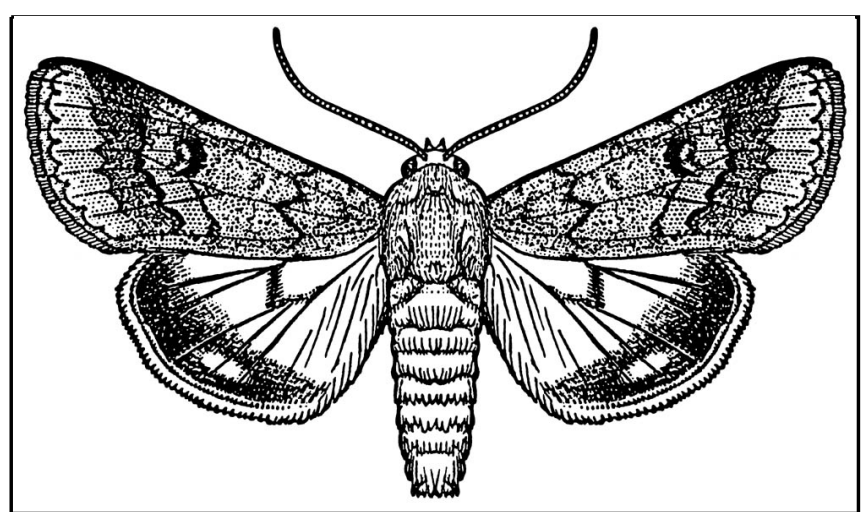

Figure 7. Corn earworm adult, Helicoverpa zea (Boddie). Credits: John L. Capinera, University of Florida

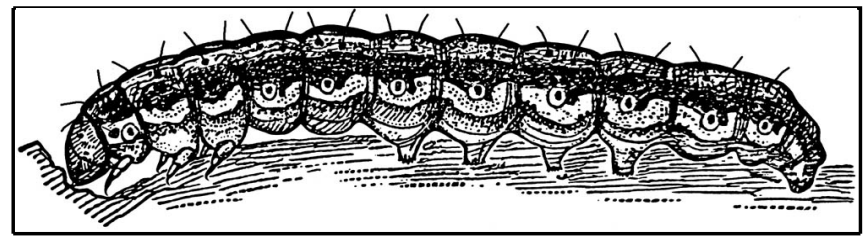

Figure 8. Corn earworm larva, Helicoverpa zea (Boddie). Credits: USDA

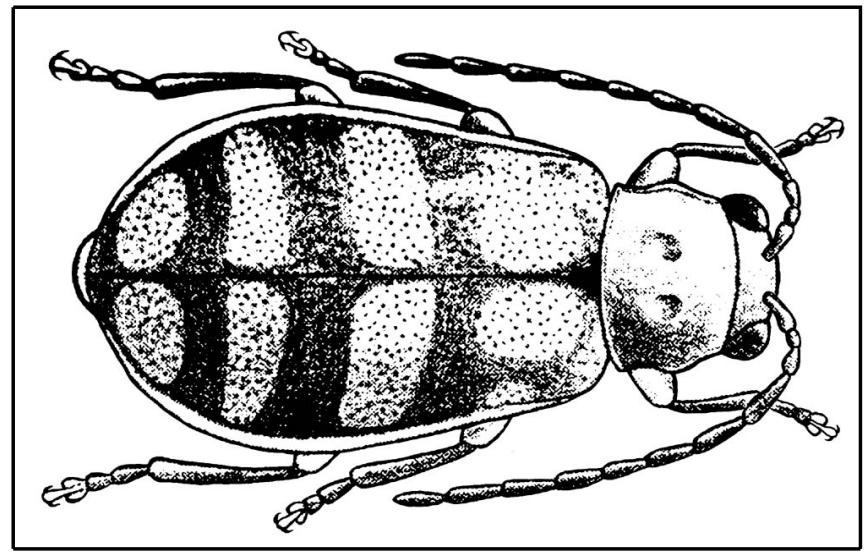

Figure 9. Banded cucumber beetle, Diabrotica balteata Leconte. Credits: John L. Capinera, University of Florida 


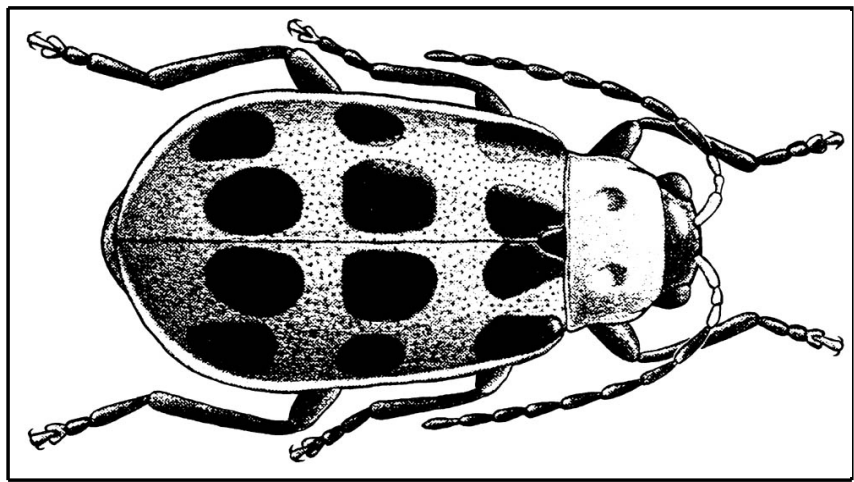

Figure 10. Spotted cucumber beetle, $D$. undecimpunctata howardi Barber. Credits: John L. Capinera, University of Florida

\section{References}

Pernezny, Ken, Gregg Nuessly, and William Stall. 2003. Integrated pest management for Florida snap beans. University of Florida, IFAS Extension. PPP37. 8 p. http://edis.ifas.ufl.edu/PP117. 


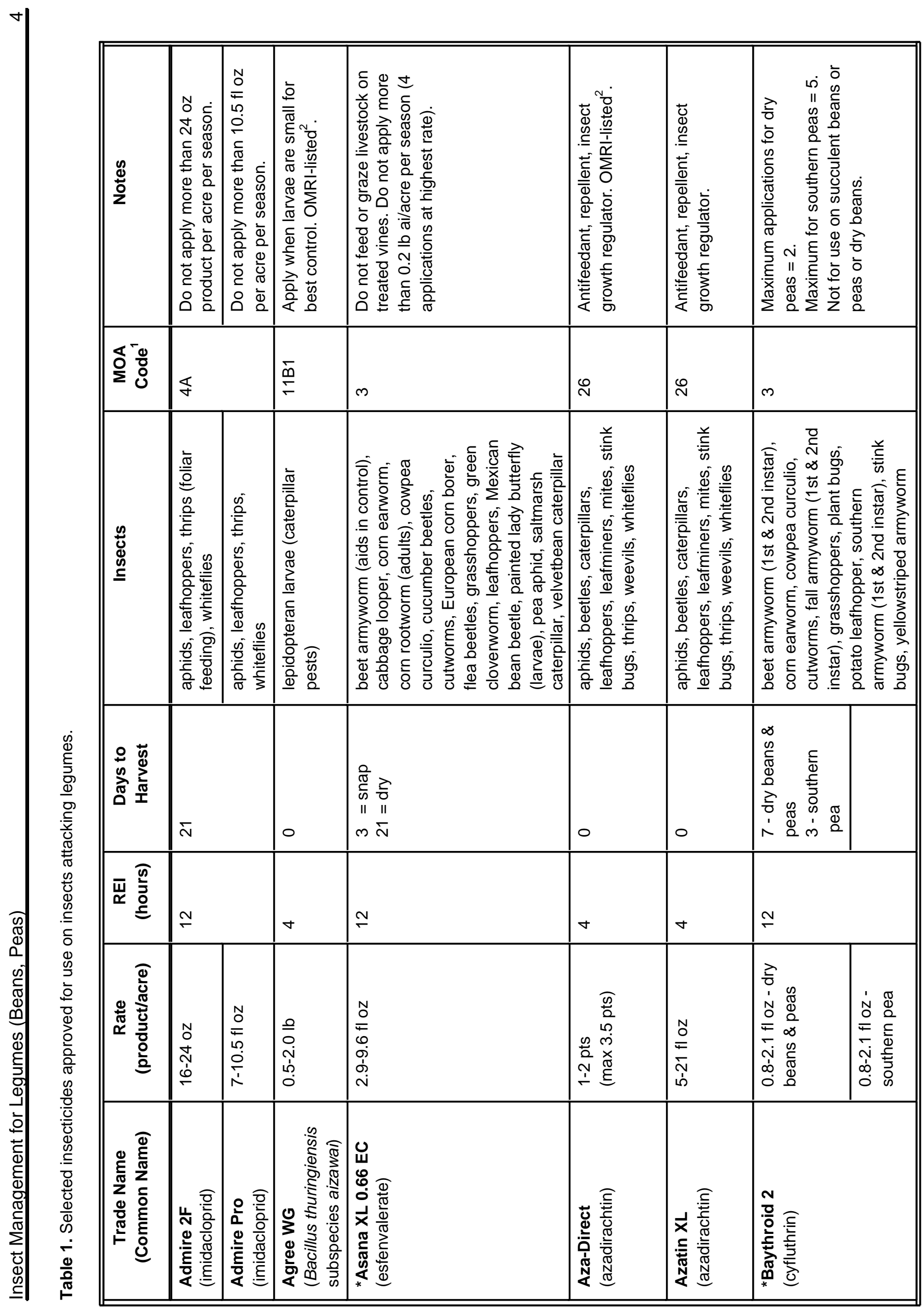




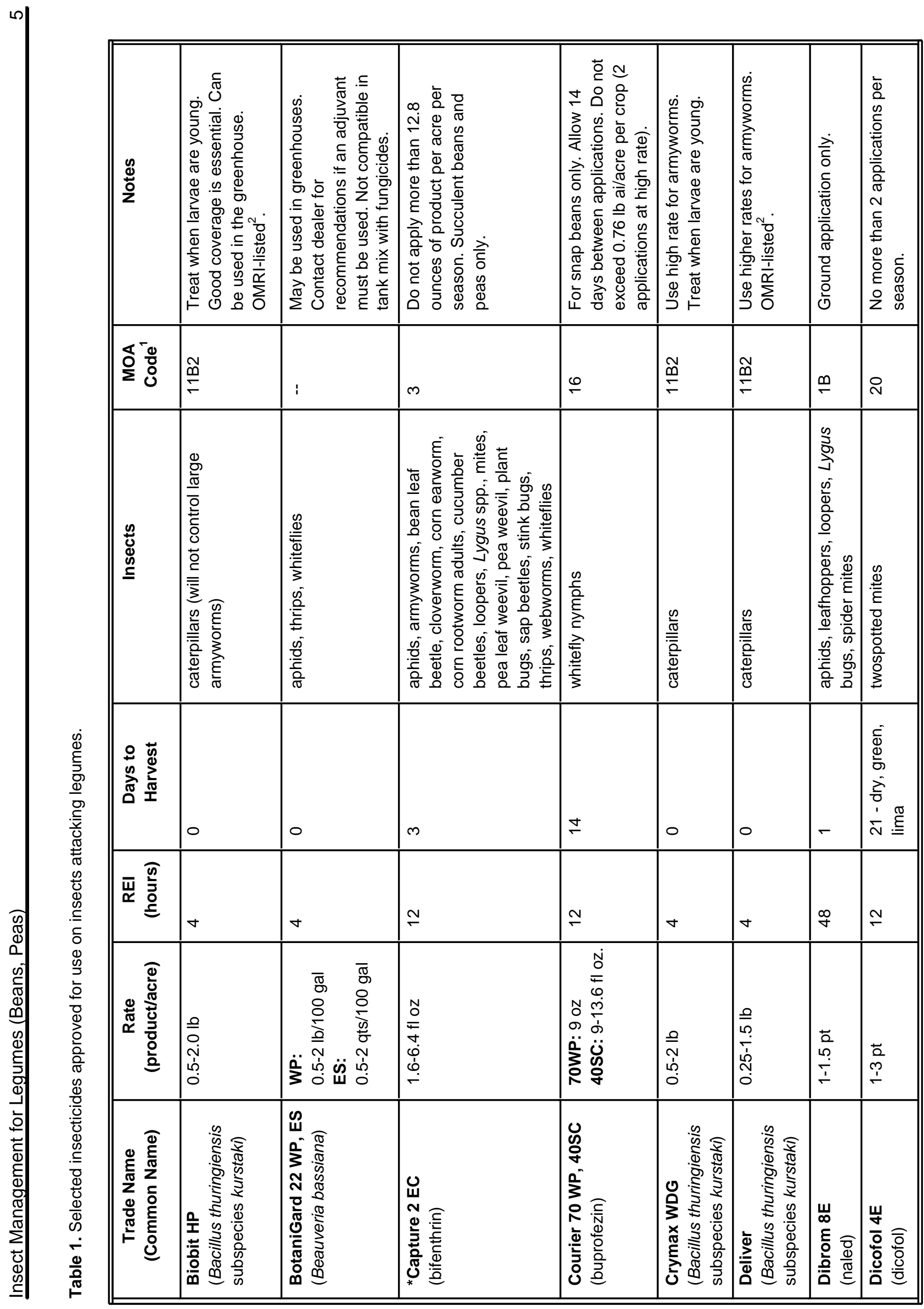




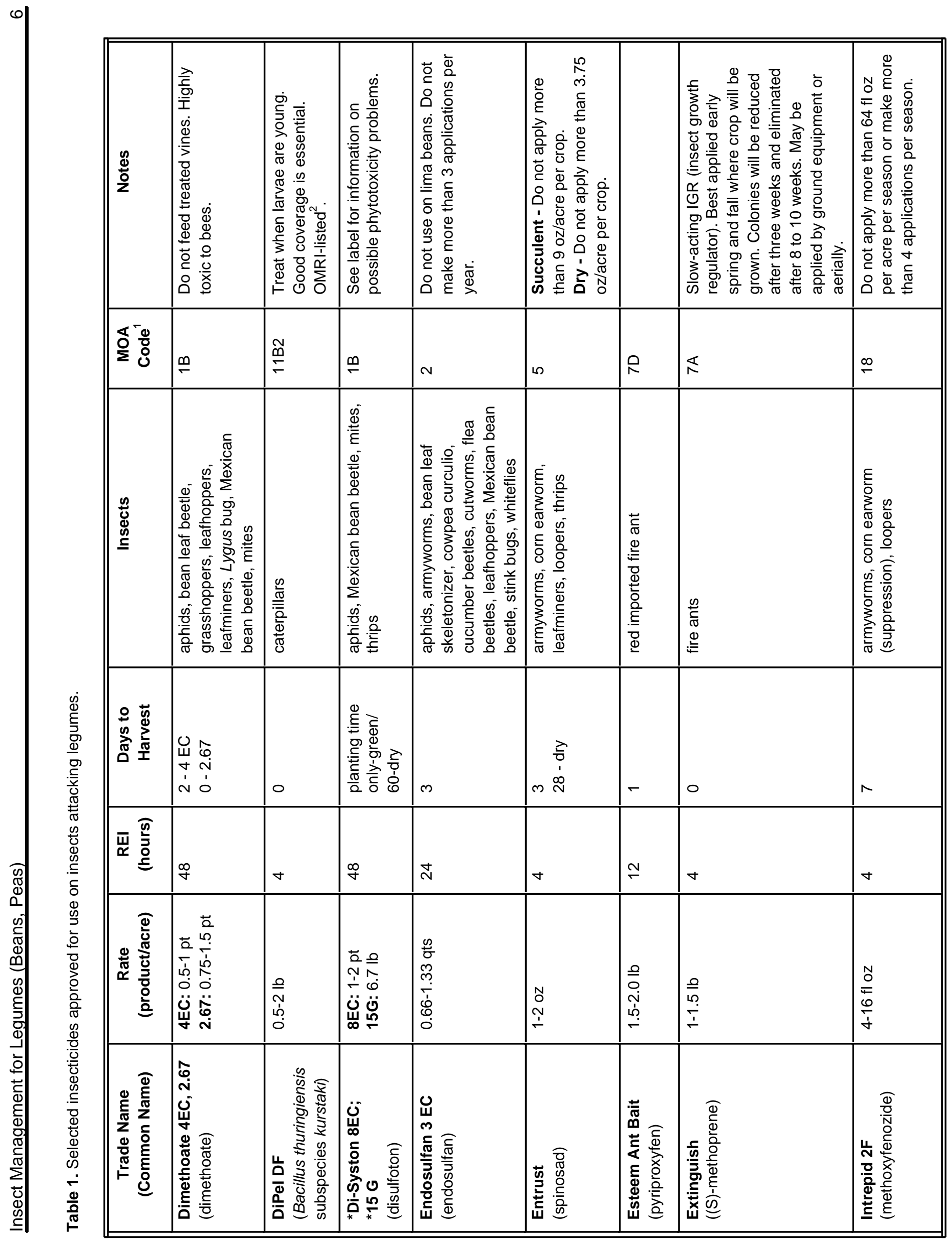




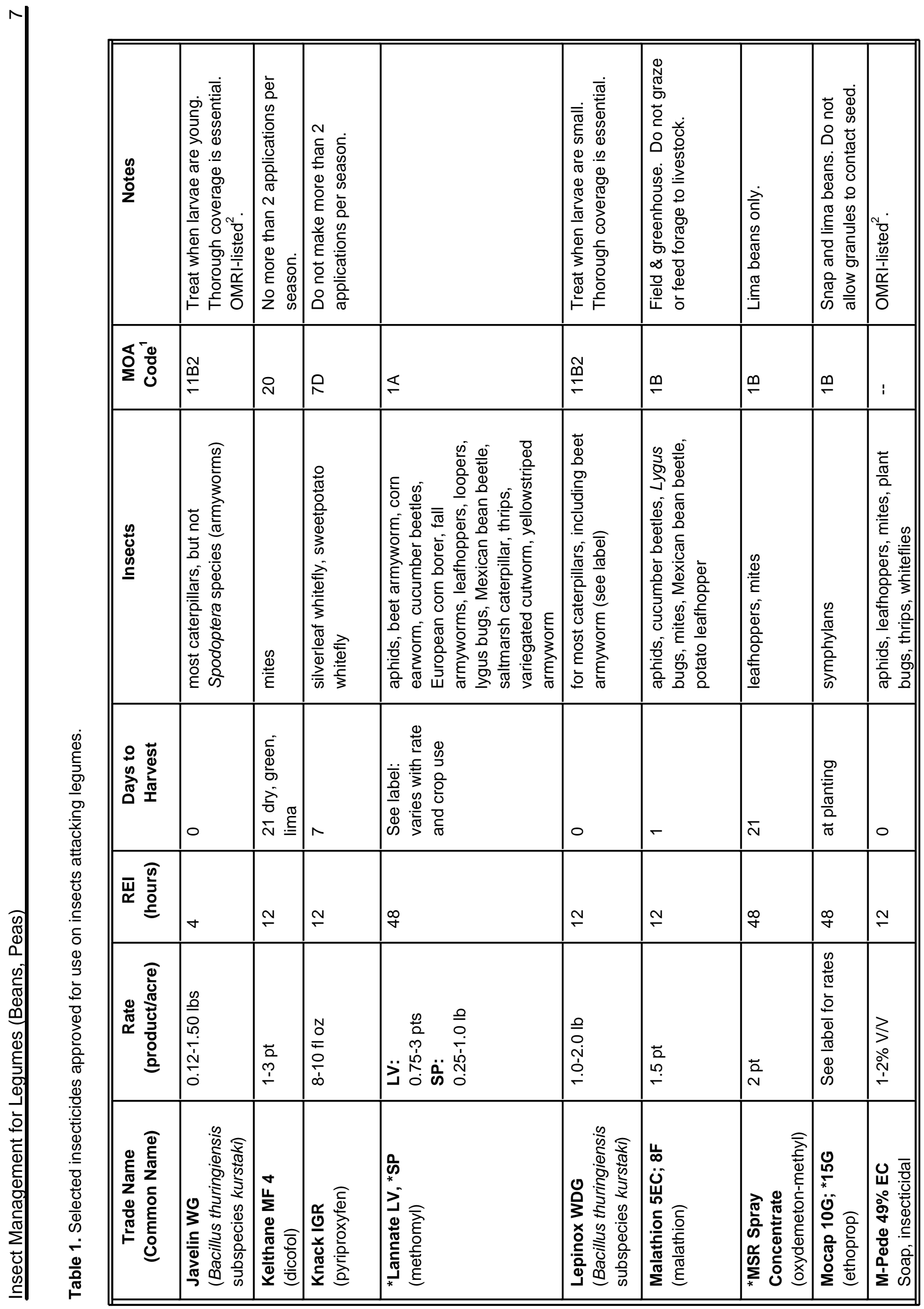




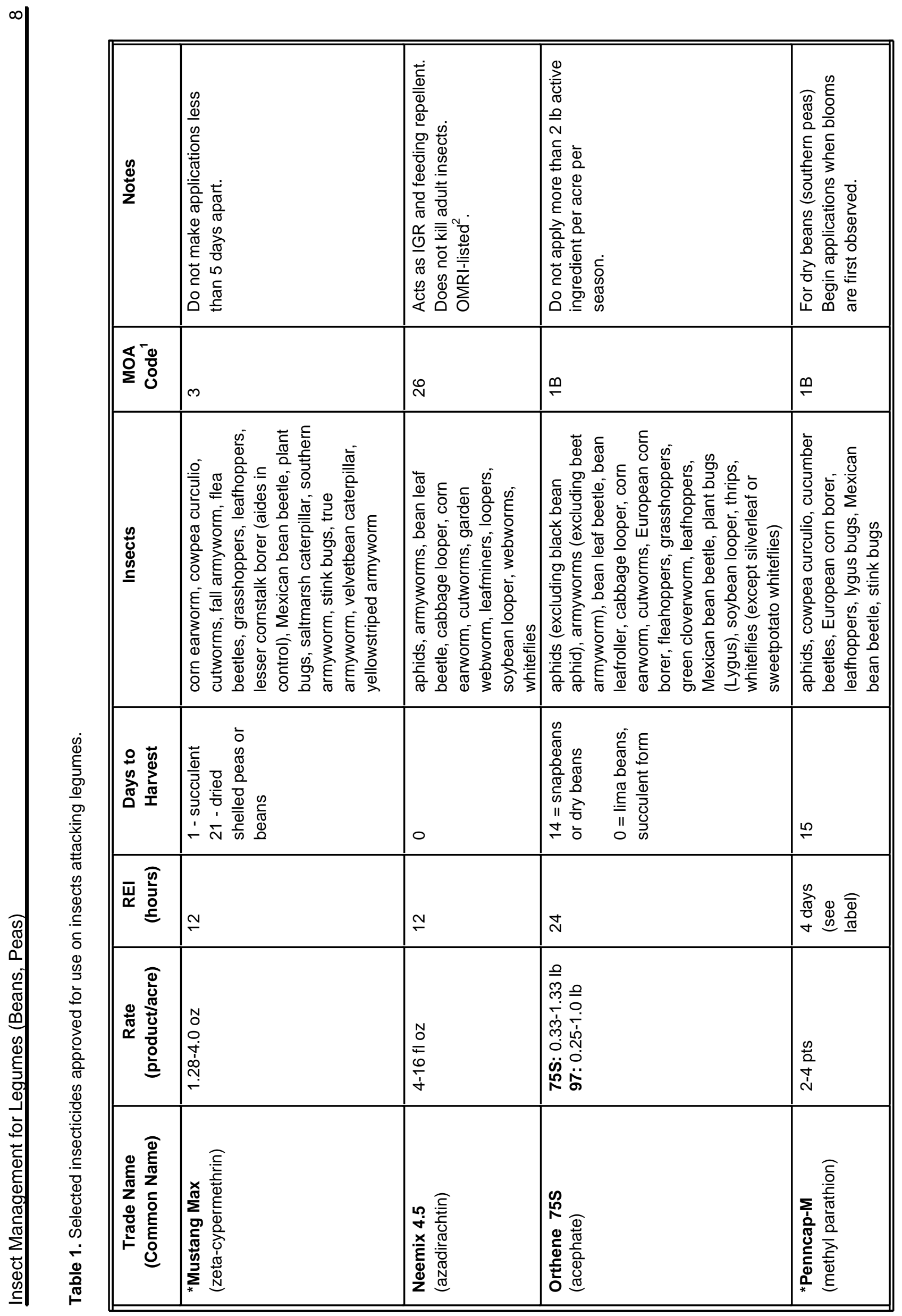




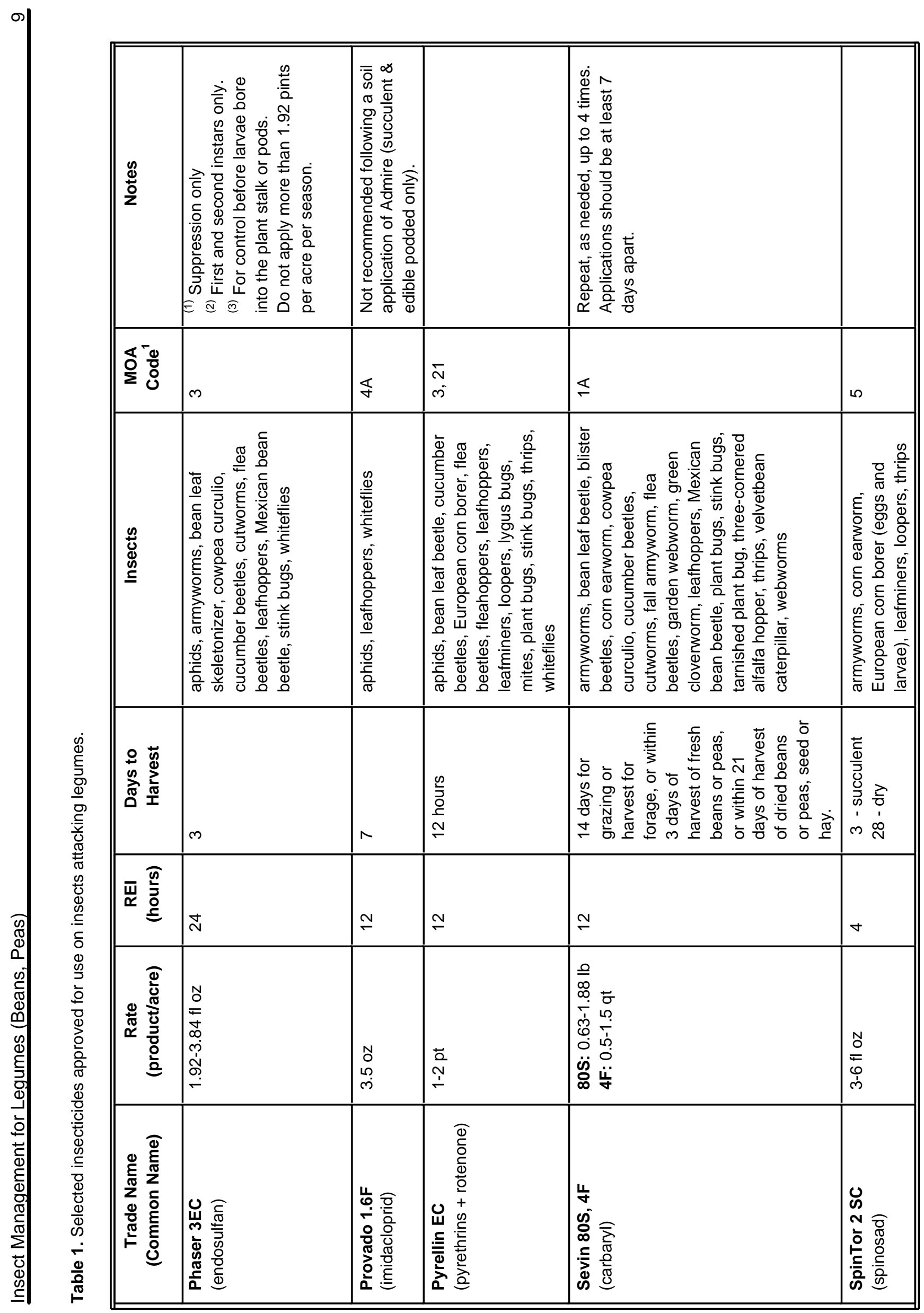




\begin{tabular}{|c|c|c|c|c|c|c|c|}
\hline & $\begin{array}{l}\mathscr{g} \\
\frac{0}{2}\end{array}$ & 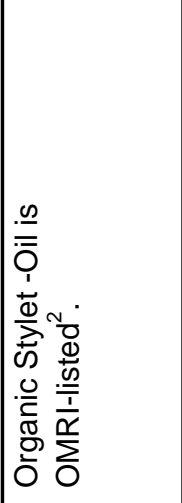 & 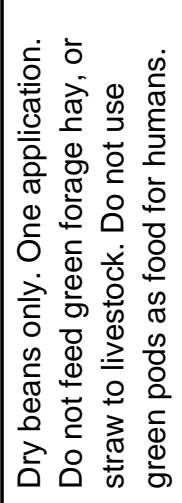 & 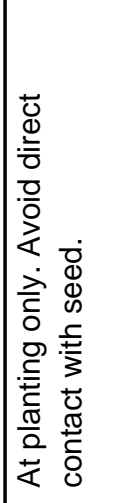 & 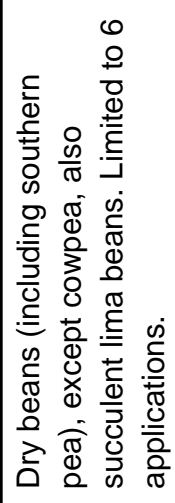 & 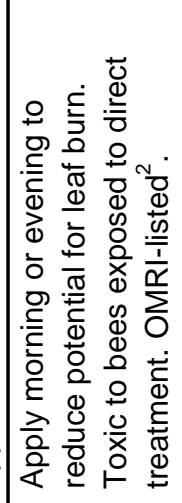 & 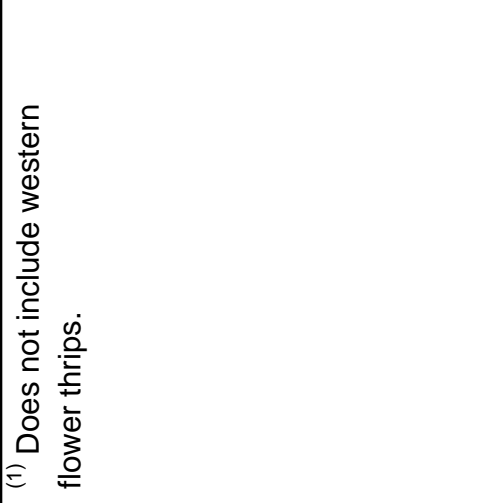 \\
\hline & 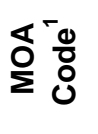 & i & $\leqslant$ & $\stackrel{\oplus}{ }$ & 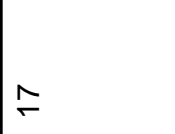 & $\stackrel{\mathscr{N}}{ }$ & m \\
\hline & 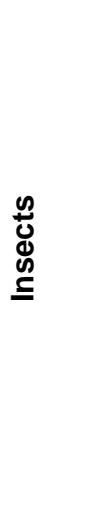 & 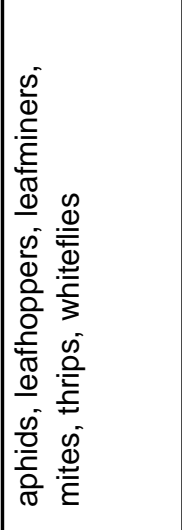 & 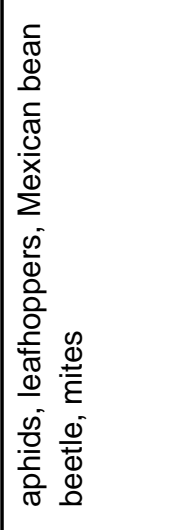 & 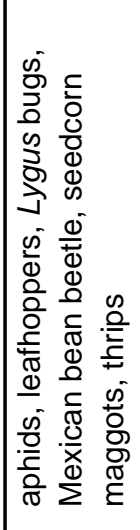 & 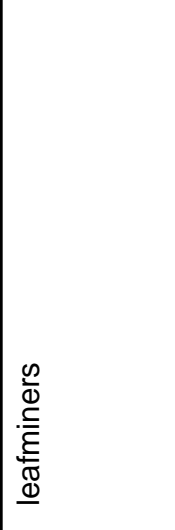 & 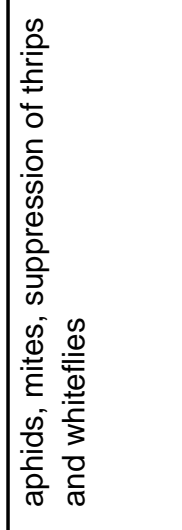 & 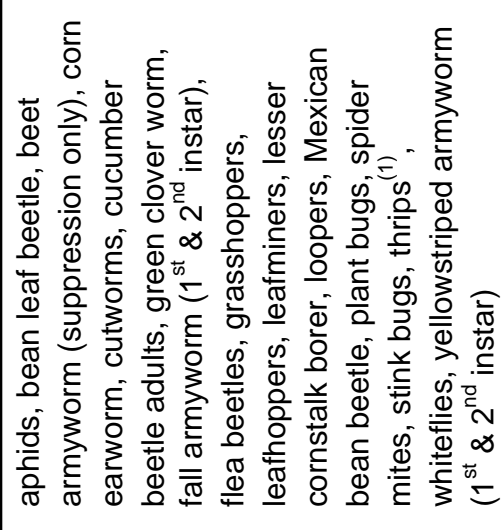 \\
\hline 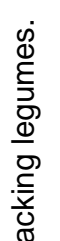 & 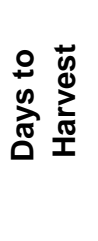 & 0 & 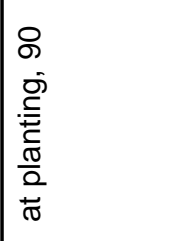 & 8 & $\wedge$ & 0 & 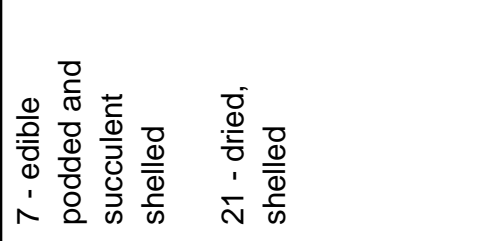 \\
\hline 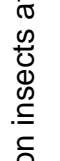 & 覂 & $\nabla$ & $\stackrel{\infty}{\sim}$ & $\stackrel{\infty}{\stackrel{\infty}{\sim}}$ & $\stackrel{\simeq}{\simeq}$ & $\nabla$ & $\stackrel{\sim}{\sim}$ \\
\hline $\begin{array}{l}0 \\
0 \\
\text { D } \\
\overline{0} \\
\frac{0}{0} \\
\Phi \\
0 \\
\frac{0}{0} \\
\frac{0}{0} \\
0 \\
0\end{array}$ & 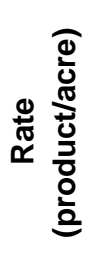 & 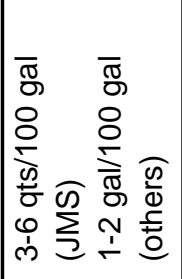 & 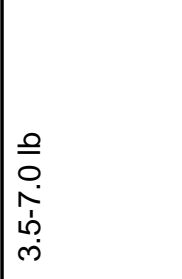 & 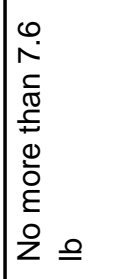 & $\begin{array}{l}N \\
0 \\
0 \\
0 \\
\end{array}$ & 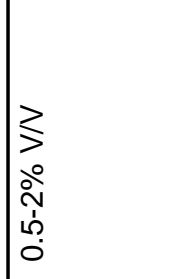 & 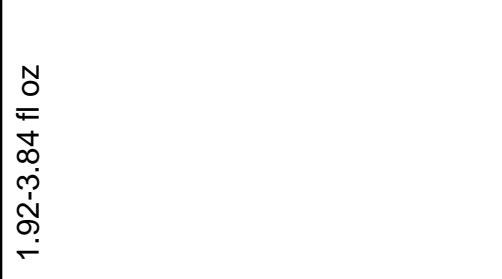 \\
\hline 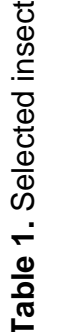 & 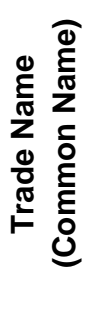 & 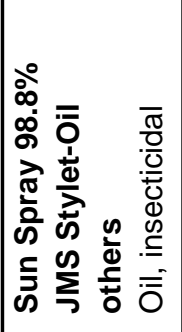 & 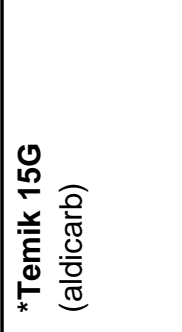 & 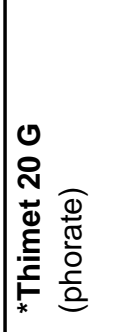 & 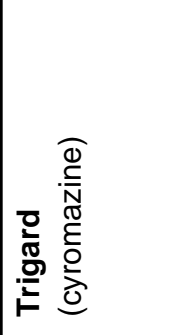 & 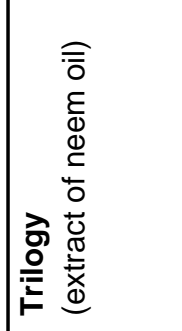 & 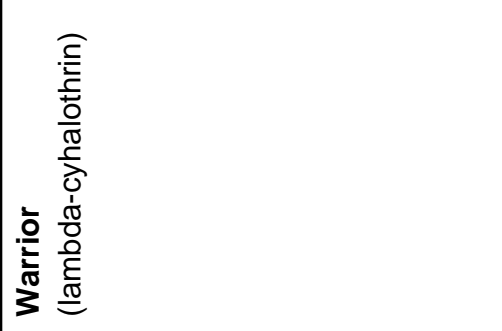 \\
\hline
\end{tabular}




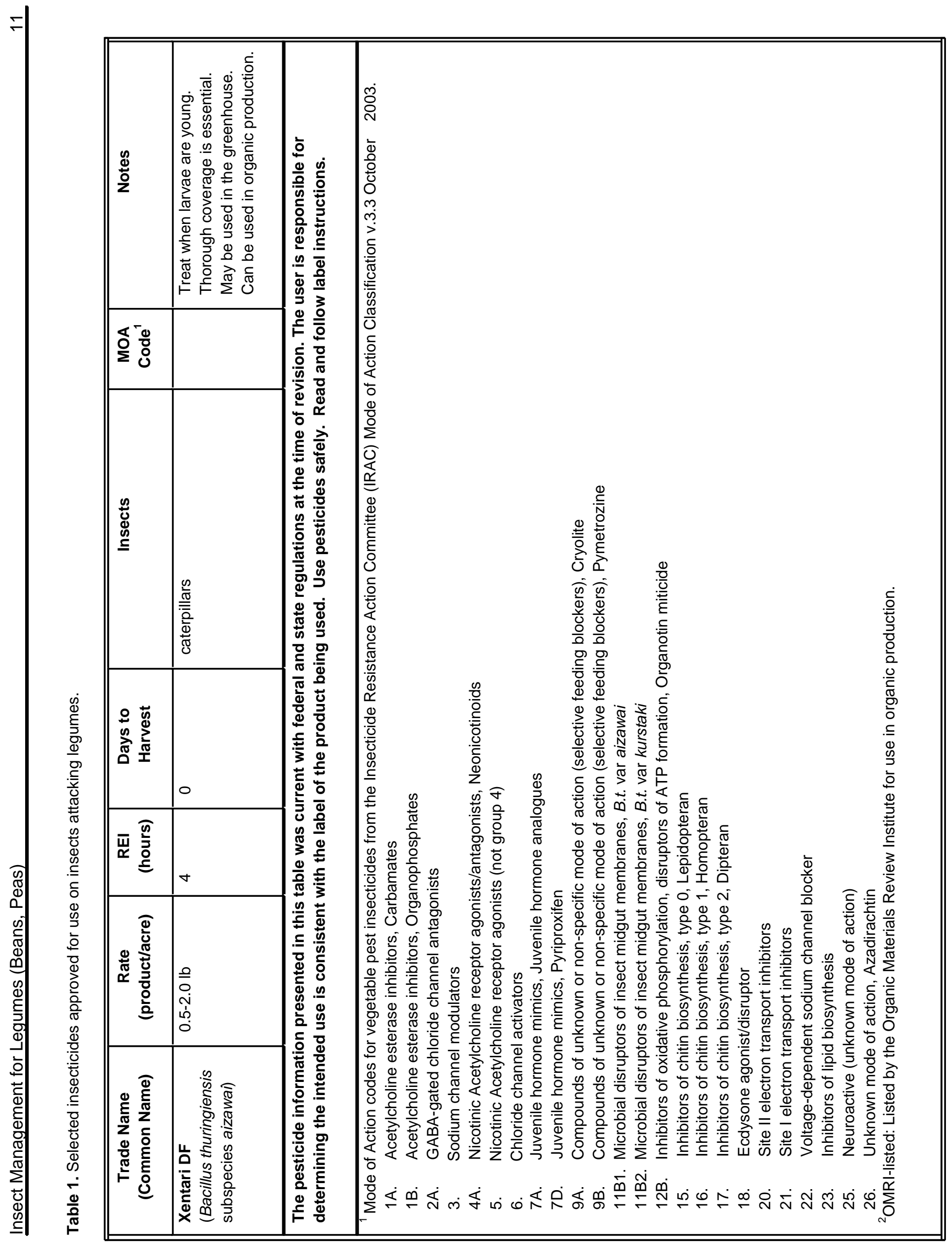

